\section{Visual and symptomatic outcome of excimer phototherapeutic keratectomy (PTK) for corneal dystrophies}

OG Stewart, P Pararajasegaram, J Cazabon and AJ Morrell
Department of Ophthalmology St James's University Hospital Beckett Street Leeds, UK

Correspondence: OG Stewart Department of Ophthalmology Clarendon Wing Leeds General Infirmary Leeds LS2 9NS, UK Tel: 01133922750 E-mail: owenstewart@ fsmail.net

\begin{abstract}
Purpose To determine the visual results and outcome of excimer laser phototherapeutic keratectomy (PTK) for corneal dystrophies. Methods Twenty-nine eyes of 19 patients who underwent excimer PTK for recurrent erosions and reduced vision due to corneal dystrophies, between February 1996 and July 1999, were reviewed. Data regarding the preoperative and postoperative best-corrected visual acuity (BCVA), change in spherical equivalent (SE), depth of excimer laser ablation, symptomatic relief, and incidence of recurrence were analysed by a retrospective chart review.

Results The range of follow-up was 12-48 months. Twenty-seven out of 29 eyes (93\%) maintained or improved BCVA. All patients (17/17) were free of symptoms of recurrent erosions although two eyes needed repeat treatment to achieve this. In those eyes undergoing PTK for reduced vision, there was a trend towards a hyperopic shift postoperatively but this was not statistically significant. Five eyes showed recurrence of the dystrophy (Reis-Bücklers (one eye), Lattice (two eyes), and Granular (two eyes)) that required repeat treatment. Two of these eyes required a single repeat PTK procedure, and three eyes underwent three repeat treatments. There were no major complications during the follow-up period. Conclusions Excimer PTK is a safe and effective procedure for relieving symptoms of recurrent erosions and improving visual acuity in patients with corneal dystrophies. Optimal visual results are achieved when treating more anterior disease. Multiple treatments are possible without significant
\end{abstract}

detrimental effects for those patients with recurrence of their dystrophy.

Eye (2002) 16, 126-131. DOI: 10.1038/

sj/EYE/6700049

Keywords: phototherapeutic keratectomy; PTK; excimer laser; corneal dystrophies; recurrent erosions

\section{Introduction}

Excimer laser phototherapeutic keratectomy (PTK) is a useful tool in the management of corneal dystrophic conditions. Visual improvement and prevention of recurrent erosions may occur following treatment. ${ }^{1}$ Anterior dystrophies are especially amenable to treatment, which is effective at clearing anterior corneal opacification, and in stabilising epithelial adherence.

Recurrent erosions often respond to conservative measures including topical lubricants, bandage contact lenses, and epithelial debridement or anterior stromal puncture. ${ }^{2}$ However, a proportion of cases will not respond or will become recurrent, especially if the primary pathological process involves instability or poor healing of the corneal epithelium as in dystrophic conditions. Over the last few years, increasing clinical evidence of the benefit of excimer PTK for recurrent corneal erosions refractory to conservative treatment, or in corneal dystrophies has been mounting. ${ }^{1,3-9}$ Despite the success of PTK, access to an excimer laser in the UK tends to be limited to large centres, and thus in many cases, repeated epithelial debridement or stromal puncture may have been performed before PTK can be carried out. 
Similarly, eyes with corneal dystrophies that cause a reduction in vision may undergo invasive procedures such as lamellar or penetrating keratoplasty before PTK. PTK is the procedure of choice if the dystrophy is limited to the anterior corneal stroma, but deeper involvement often requires more definitive treatment. It has been noted that any invasive procedure can cause an acceleration of the dystrophy. However, once a cornea has been grafted, the dystrophy often appears to be more anteriorly situated ${ }^{9}$ and therefore more likely to be amenable to PTK. Multiple treatments are possible and visual rehabilitation tends to be fairly rapid. It may therefore be possible to avoid or defer procedures such as lamellar or penetrating keratoplasty, with their associated greater morbidity.

\section{Materials and methods}

We performed a retrospective study of 36 consecutive PTK procedures on 29 eyes of 19 patients. Data collected included pre- and postoperative bestcorrected visual acuity (BCVA), pre- and postoperative spherical equivalent (SE), the depth of ablation, symptomatic relief from recurrent corneal erosions, and the incidence of recurrence of erosions or of the dystrophy. The pre- and postoperative spherical equivalent was subject to statistical analysis using the paired $t$-test, and possible correlation between depth of laser ablation and change in spherical equivalent was assessed by calculating the correlation coefficient, $r$.

All of the patients were collected from the cornea and external eye disease clinic at St James's University Hospital, Leeds, which is a tertiary referral centre; and were treated by a single surgeon (AJM). There were 11 females and eight males. Nine patients had unilateral treatment and 10 had bilateral treatment. The age range of the patients was between 26 and 74 years (mean 48.6 years). Follow-up ranged from 12 months to 48 months (mean 22.3 months).

The indication for treatment was recurrent erosions or epithelial instability in 17 eyes, reduced vision in 12 eyes and both in two eyes. All eyes with recurrent erosions had failed to respond to medical therapy, therapeutic contact lenses, epithelial debridement or anterior stromal puncture. These patients had endured recurrent problems over a significant period of time prior to the availability of the excimer laser in Yorkshire. The preoperative diagnosis is summarised in Figure 1.

Seven eyes of six patients had undergone previous penetrating keratoplasty. Five of these had a single graft (two with Lattice dystrophy and three with Granular dystrophy) and two eyes of one patient had
2

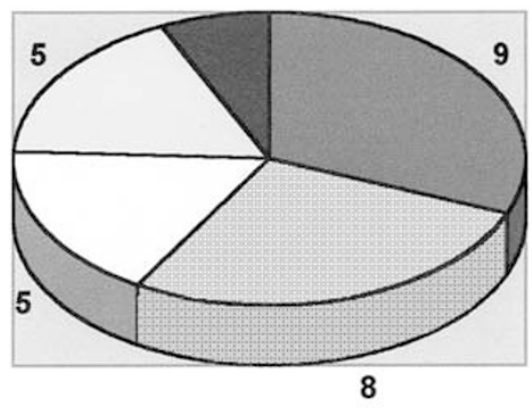

Figure 1 Preoperative diagnosis.

undergone three grafts each (patient 12-Granular dystrophy).

The Technolas $116193 \mathrm{~nm}$ excimer laser (Bausch \& Lomb Surgical, Inc, USA) was used for all but one treatment, for which the Technolas 217 (Bausch \& Lomb) was used. Whilst using the 116, the number of pulses was set according to the depth of ablation desired, with 1 pulse giving an approximate depth of $0.25 \mu \mathrm{m}$. The ablation depth was calculated from the number of pulses used. The central $7 \mathrm{~mm}$ of cornea was treated with multiple overlapping peripheral $3 \mathrm{~mm}$-diameter satellite areas. With the Technolas 217, the depth was preset and the central $7 \mathrm{~mm}$ was treated with a blending of ablative depth out to $10 \mathrm{~mm}$.

All treatments were centred on the visual axis. After instillation of topical local anaesthetic, a lid speculum was inserted and the patient asked to fixate on the fixation light. Mechanical epithelial debridement was performed in the majority of cases. If the surface was very irregular, methylcellulose was used as a masking agent to smooth out surface irregularities before ablation.

The depth of ablation varied according to the primary pathology and to the purpose of treatment. Per-operative slit lamp examination was used to determine whether the desired depth had been reached for cases with stromal dystrophies. In four eyes of two patients (patients 10 and 12), PTK was performed in an 
effort to both improve visual acuity and to treat recurrent erosions (Table 1 ).

Postoperative treatment consisted of immediate application of topical chloramphenicol $0.5 \%$ and voltarol $0.1 \%$ prior to instillation of a bandage contact lens. Follow-up occurred at $48 \mathrm{~h}$, and then at 1, 4, and 12 weeks. Following this, review generally occurred at 6 monthly intervals. The bandage contact lens was usually removed at $48 \mathrm{~h}$, and the antibiotic drops were continued until full epithelial healing had occurred.

Topical lubricants (Viscotears, Alcon Labs) were used for at least 3 months postoperatively.

\section{Results}

Fifteen eyes of 11 patients underwent PTK for recurrent erosions or epithelial instability alone. Thirteen out of 15 (87\%) procedures were deemed to have been successful, whilst two eyes required repeat treatment (Table 2).

Of the eyes treated for reduced vision alone, all 10
Table 2 Times of recurrence of dystrophy following PTK

\begin{tabular}{lcc}
\hline Dystrophy & \multicolumn{1}{c}{$\begin{array}{c}\text { Time of recurrence following PTK } \\
\text { (months) }\end{array}$} \\
\cline { 2 - 3 } & $\begin{array}{l}\text { Following } 1 s t \\
\text { procedure }\end{array}$ & $\begin{array}{l}\text { Following 2nd } \\
\text { procedure }\end{array}$ \\
\hline Reis-Bücklers & 10 & - \\
Lattice & 20 & 13 \\
Granular & 35 & 7 \\
Map-dot-fingerprint & 11 & - \\
\hline
\end{tabular}

eyes maintained or improved Snellen BCVA following PTK, with a mean improvement of BCVA of 1.85 lines. Four eyes of two patients were treated for a combination of recurrent erosions and for reduced vision. These treatments were successful at abolishing further erosions but two eyes of one patient with granular dystrophy subsequently lost BCVA due to recurrence of the dystrophy and further treatment is awaited.

Table 1 Indications for treatment, depth of ablation, and the changes in BCVA and SE following PTK (at 12 month follow-up)

\begin{tabular}{|c|c|c|c|c|c|c|c|}
\hline \multicolumn{2}{|c|}{ Patient $\mathcal{E}$ eye } & Diagnosis & $\begin{array}{c}\text { Indication for } \\
\text { PTK }\end{array}$ & $\begin{array}{c}\text { Preoperative } \\
\text { BCVA }\end{array}$ & $\begin{array}{c}\text { Postoperative } \\
\text { BCVA }\end{array}$ & $\begin{array}{c}\text { Depth of ablation } \\
(\mu \mathrm{m})\end{array}$ & Change in SE \\
\hline \multirow[t]{2}{*}{1} & $\mathrm{R}$ & MDF & RCE & $6 / 6$ & $6 / 5$ & 7 & -0.75 \\
\hline & $\mathrm{L}$ & & & $6 / 9$ & $6 / 5$ & 7 & 0.00 \\
\hline \multirow[t]{2}{*}{2} & $\mathrm{R}$ & Reis-Bücklers & $\mathrm{RCE}$ & $6 / 36$ & $6 / 36$ & $112^{*}$ & -0.50 \\
\hline & $\mathrm{L}$ & & & $6 / 18$ & $6 / 12$ & 30 & +0.25 \\
\hline 3 & & MDF & $\mathrm{RCE}$ & $6 / 6$ & $6 / 4$ & 7 & 0.00 \\
\hline 4 & & MDF & RCE & $6 / 6$ & $6 / 5$ & 7 & +0.62 \\
\hline 5 & & MDF & RCE & $6 / 18$ & $6 / 5$ & 7 & +1.62 \\
\hline \multirow[t]{2}{*}{6} & $\mathrm{R}$ & Fuchs' & RCE & $6 / 12$ & $6 / 9$ & 10 & -0.37 \\
\hline & $\mathrm{L}$ & & & $6 / 18$ & $6 / 9$ & 10 & -1.00 \\
\hline 7 & & MDF & RCE & $6 / 12$ & $6 / 6$ & 7 & +2.00 \\
\hline 8 & & MDF & RCE & $6 / 5$ & $6 / 5$ & 7 & +0.50 \\
\hline 9 & & Lattice & $\mathrm{RCE}$ & $6 / 9$ & $6 / 6$ & 23 & -1.50 \\
\hline \multirow[t]{2}{*}{10} & $\mathrm{R}$ & Reis-Bücklers & $\mathrm{RCE}+\mathrm{RVA}$ & $6 / 36$ & $6 / 12$ & 51 & -0.12 \\
\hline & $\mathrm{L}$ & & $\mathrm{RCE}+\mathrm{RVA}$ & $6 / 18$ & $6 / 18$ & $100^{*}$ & +0.12 \\
\hline \multirow[t]{2}{*}{11} & $\mathrm{R}$ & MDF & $\mathrm{RCE}$ & $6 / 18$ & $6 / 6$ & 7 & +0.12 \\
\hline & $\mathrm{L}$ & & & $6 / 9$ & $6 / 6$ & 7 & -0.67 \\
\hline \multirow[t]{2}{*}{12} & $\mathrm{R}$ & Granular & $\mathrm{RCE}+\mathrm{RVA}$ & $3 / 24$ & $3 / 60$ & 62 & +7.00 \\
\hline & $\mathrm{L}$ & & $\mathrm{RCE}+\mathrm{RVA}$ & $6 / 24$ & $6 / 36$ & 88 & -1.00 \\
\hline \multirow[t]{2}{*}{13} & $\mathrm{R}$ & Reis-Bücklers & RVA & $6 / 12$ & $6 / 6$ & $65^{* *}$ & +1.12 \\
\hline & $\mathrm{L}$ & & & $6 / 12$ & $6 / 6$ & $68^{*}$ & -0.25 \\
\hline 14 & & Lattice & RCE & $6 / 18$ & $6 / 12$ & 7 & +1.75 \\
\hline 15 & & Granular & RVA & $6 / 36$ & $6 / 12$ & 29 & -0.50 \\
\hline 16 & & Lattice & RVA & $6 / 18$ & $6 / 9$ & 76 & -3.00 \\
\hline \multirow[t]{2}{*}{17} & $\mathrm{R}$ & Lattice & RVA & $6 / 36$ & $6 / 6$ & $137^{* *}$ & +3.00 \\
\hline & $\mathrm{L}$ & & & $6 / 36$ & $6 / 18$ & $125 / 60 / 17$ & +5.25 \\
\hline \multirow[t]{2}{*}{18} & $\mathrm{R}$ & Granular & RVA & $6 / 24$ & $6 / 18$ & $100 / 60^{*} / 75^{*}$ & +1.12 \\
\hline & $\mathrm{L}$ & & & $6 / 36$ & $6 / 24$ & $100 / 85^{*} / 75$ & +4.25 \\
\hline \multirow[t]{2}{*}{19} & $\mathrm{R}$ & Reis-Bücklers & RVA & $6 / 18$ & $6 / 9$ & 30 & -0.37 \\
\hline & $\mathrm{L}$ & & & $6 / 24$ & $6 / 12$ & 20 & -0.37 \\
\hline
\end{tabular}

RCE, recurrent erosions. RVA, reduced visual acuity. ${ }^{* W i t h}$ methylcellulose. ${ }^{* *}$ Simultaneous PARK. 
Improvement in vision was maintained during the study period in nine of the 14 eyes treated for visual reasons. Five eyes developed recurrence of the dystrophy that required further PTK. The times of recurrence following PTK are summarised in Table 2.

Overall, 27 out of 29 eyes (93\%) maintained or improved BCVA following PTK for their dystrophic condition at a 12-month follow-up (Figure 2).

Refractive changes postoperatively were variable. Fourteen eyes showed a hyperopic shift (48\%) and 13 eyes showed a myopic shift (45\%) (Figure 3). In the group of eyes treated for reduced vision, the preoperative mean SE was $-1.33 \mathrm{D}(\mathrm{SD} \pm 5.44)$, and the mean postoperative SE was $-0.57 \mathrm{D}(\mathrm{SD} \pm 4.02)$. Although this reveals a trend towards a hyperopic shift, the difference was not significant $(P>0.5)$. Patient 12 experienced a 7.00 D hyperopic shift with only $62 \mu \mathrm{m}$ ablation in one eye. However, the accuracy of the preoperative $(-23 \mathrm{D})$, and the postoperative refractions $(-18.00 /+6.00 \times 130)$, in this patient must be brought into question as he had previously undergone three penetrating grafts, and had advanced recurrence of granular dystrophy at the time of PTK. Likewise, there was no significant difference between the preoperative mean SE in the recurrent erosions group $(-0.25 \mathrm{D}(\mathrm{SD} \pm 5.01))$ and the postoperative $\mathrm{SE}$ $(-0.20 \mathrm{D}(\mathrm{SD} \pm 5.07))$. In those patients undergoing PTK for visual reasons, there was a positive correlation between the depth of ablation and a hyperopic shift ( $r$ $=0.48$ ). Two eyes (patients 13 and 17) underwent

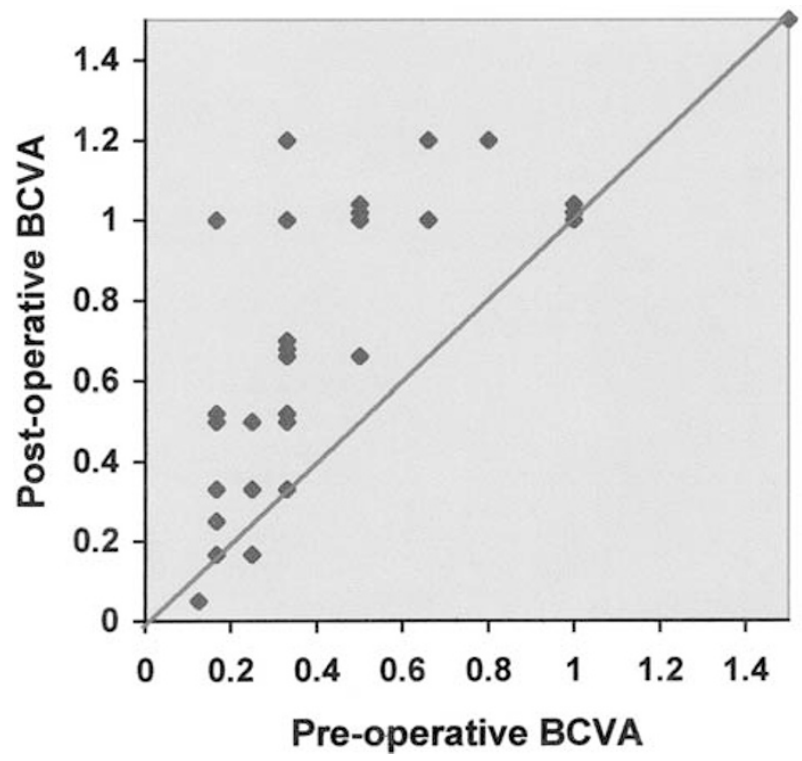

Figure 2 BCVA before and after PTK (12 months follow-up).

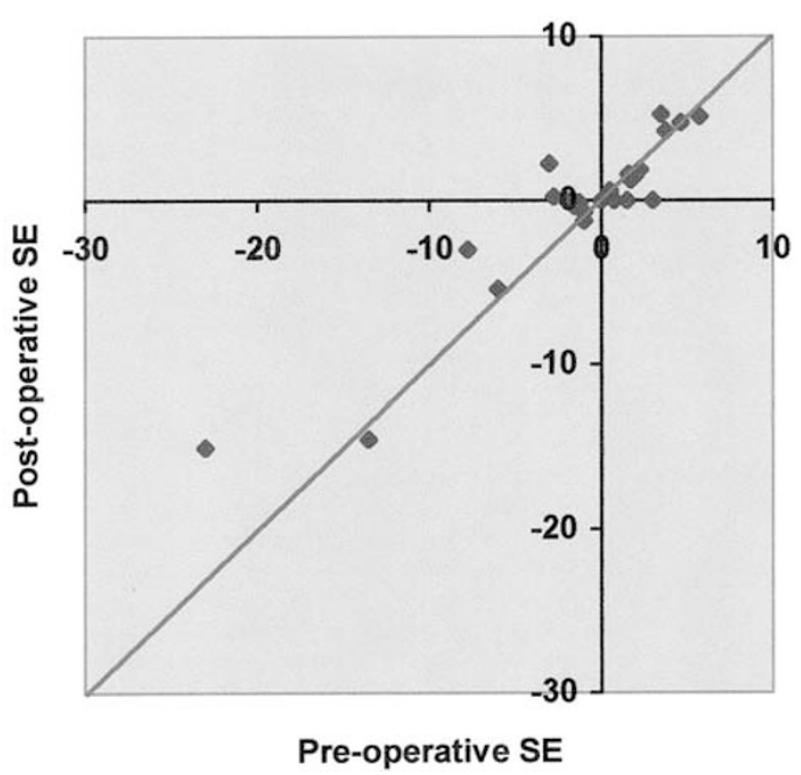

Figure 3 Pre- and postoperative spherical equivalent (all patients).

simultaneous photo-astigmatic refractive keratectomy (PARK).

There were no major complications seen in this series of patients. Three eyes with granular dystrophy developed mild subepithelial fibrosis. Postoperative pain was short-lived and helped with therapeutic contact lenses and topical NSAIDs.

Table 1 summarises the indication for treatment, the depth of ablation, and the changes in BCVA and SE following PTK.

\section{Discussion}

Excimer PTK has been used for over a decade in the management of a number of various corneal conditions with excellent results. Attempts may be made to improve epithelial stability or to improve visual acuity. ${ }^{1,3-9}$ It may also be combined with refractive treatment to reduce ametropia or astigmatism. In addition, PTK can be safely performed in eyes which have previously undergone penetrating keratoplasty. It is a quick procedure that may be performed under topical anaesthesia on an out-patient basis. For all these reasons, it is rapidly becoming the procedure of choice in a number of clinical situations.

Based on the experiences of treating recurrent corneal erosions in this series of patients, excimer PTK offers a safe and more effective form of treatment than other modalities of treatment, namely anterior stromal 
puncture $^{2}$ or epithelial debridement. In all, 16/18 eyes $(89 \%)$ were free of recurrence from one procedure during the follow-up period. These figures include 9/10 eyes (90\%) with MDF dystrophy. One eye with this condition required retreatment due to significant recurrence of erosions 9 months following the first procedure. This compares well with a larger series of patients with this dystrophy by Cavanaugh et al where they report $86.6 \%$ success rate after a single procedure; ${ }^{3}$ and mirrors the findings by Örndahl, in which $90 \%$ of eyes were recurrence free. ${ }^{4}$ These figures also suggest that PTK for recurrent erosions secondary to other corneal dystrophies is extremely effective. Anterior stromal conditions such as Reis-Bücklers, lattice, and granular dystrophies all predispose to recurrent erosions, and were all effectively treated in this series, as were two eyes with epithelial instability resulting from corneal oedema secondary to Fuch's endothelial dystrophy. PTK may be used in this condition as an interim measure for painful erosions prior to penetrating keratoplasty, or if surgical correction is not indicated. Providing that the stromal oedema is not too severe, increased epithelial stability may be achieved with ablation depths similar to those used in MDF dystrophy. Thomann et al has also previously described the effectiveness of PTK in bullous keratopathy. ${ }^{5}$ Excimer PTK is an ideal modality of treatment for MDF dystrophy as limited depth of ablation is required for this superficial condition. Recurrence rates are extremely low, and improvement of BCVA may be achieved, possibly due to reducing the induced irregular astigmatism from epithelial irregularity. ${ }^{3}$ Corneal scarring or haze rarely occurs and significant induced hyperopia tends not to occur unless deeper ablation is performed. In this series, there was no significant refractive change post-PTK in the recurrent erosion group.

Treatment performed for reduced visual acuity was largely successful in clearing central corneal opacification due to dystrophic changes. Mild subepithelial fibrosis developed in three eyes with granular dystrophy, but visual improvement and rehabilitation was rapid in the majority of cases. The time until recurrence was variable in this series. Five out of 14 eyes (36\%) subsequently required further PTK. Dinh et al reported variable rates of recurrence between different dystrophies, with granular dystrophy tending to be the slowest to re-occur. ${ }^{10}$ Obviously, as time goes on, the likelihood of visually significant recurrence increases irrespective of the primary condition. However, PTK can be safely repeated without significant adverse effects. Repeat procedures were well tolerated, even in previously grafted eyes. Repeat treatment potentially increases the chance of hyperopic shift postoperatively due to corneal flattening, but this was not seen to any significant degree. Refractive changes following PTK were variable, and a mild myopic shift was seen in $45 \%$ of the patients. O'Brart et al suggested that this may represent a slight steepening of the cornea due to peripheral ablation, ${ }^{6}$ but neither they nor ourselves could demonstrate this topographically. Other factors involved may be epithelial remodelling, concurrent ocular pathology eg early nuclear sclerosis, or inaccuracy of the preoperative refraction due to severe corneal scarring.

Pachymetry measurements between treatments did not seem to reveal a significant reduction in corneal thickness in two of the eyes undergoing four PTK procedures. It could be hypothesised that, with time, there is almost complete redeposition of dystrophic material in the ablated area.

It has previously been reported that recurrence of a dystrophy following lamellar or penetrating keratoplasty tends to occur centrally and at a superficial level. Early recurrence of granular dystrophy ${ }^{11}$ has been noted to be central and epithelial with the occasional vortex pattern, and only later involves deeper layers. It tends to be observed in most grafts within 36 months after surgery. Likewise, the recurrence of lattice dystrophy is characterised by subepithelial opacities and/or anterior stromal haze. Recurrence of Reis-Bücklers dystrophy tends to occur diffusely at the level of Bowman's membrane and may become visually significant earlier than other dystrophies. ${ }^{10}$ Therefore, the superficial level, and the central location of recurrence of a corneal dystrophy mean that PTK is the procedure of choice in these situations.

In summary, we have found excimer PTK to be a safe and extremely effective procedure in the management of recurrent corneal erosions and reduced visual acuity due to corneal dystrophies. It is repeatable and can be safely performed in previously grafted eyes. The use of PTK may defer or abolish the need for more interventional modalities of treatment such as lamellar or penetrating keratoplasty, which can only be beneficial in this time of decreasing availability of corneal donor tissue in the UK.

\section{References}

1 Örndahl MJF, Fagerholm PP. Treatment of corneal dystrophies with phototherapeutic keratectomy. J Refract Surg 1998; 14: 129-135.

2 McLean EN, MacRae SM, Rich LF. Recurrent erosion. Treatment by anterior stromal puncture. Ophthalmology 1986; 93: 784-788. 
3 Cavanaugh TB, Lind DM, Cutarelli PE et al. Phototherapeutic keratectomy for recurrent erosion syndrome in anterior basement membrane dystrophy. Ophthalmology 1999; 106: 971-976.

4 Örndahl MJF, Fagerholm PP. Phototherapeutic keratectomy for Map-Dot-Fingerprint corneal dystrophy. Cornea 1998; 17: 595-599.

5 Thomann U, Meier-Gibbons F, Schipper I.

Phototherapeutic keratectomy for bullous keratopathy. $\mathrm{Br}$ J Ophthalmol 1995; 79: 335-338.

6 O'Brart DPS, Kerr Muir MG, Marshall J. Phototherapeutic keratectomy for recurrent corneal erosions. Eye 1994; 8: 378-383.

7 Morad Y, Haviv D, Zadok D et al. Excimer laser phototherapeutic keratectomy for recurrent corneal erosion. J Cataract Refract Surg 1998; 24: 451-455.
8 Lohmann CP, Sachs H, Marshall J et al. Excimer laser phototherapeutic keratectomy for recurrent erosions: a clinical study. Ophthal Surg Lasers 1996; 27: 768-772.

9 Dausch D, Landesz M, Klein R et al. Phototherapeutic keratectomy in recurrent corneal epithelial erosion. Refract Corneal Surg 1993; 9: 419-424.

10 Dinh R, Rapuano CJ, Cohen EJ et al. Recurrence of corneal dystrophy after excimer laser phototherapeutic keratectomy. Ophthalmology 1999; 106(8): 1490-1497.

11 Lyons CJ, McCartney AC, Kirkness CM et al. Granular corneal dystrophy. Visual results and pattern of recurrence after lamellar or penetrating keratoplasty. Ophthalmology 1994; 101: 1812-1817. 